\title{
Development of Itraconazole-Loaded Polymeric Nanoparticle Dermal Gel for Enhanced Antifungal Efficacy
}

\author{
Hoang Nhan Ho $\mathbb{D},{ }^{1}$ Thien Giap Le $\mathbb{D}^{2,3}$ Thi Thanh Tuyen Dao, ${ }^{3}$ Thi Ha Le ${ }^{\left(\mathbb{D},{ }^{3}\right.}$ \\ Thi Thanh Hai Dinh, ${ }^{4}$ Dang Hoa Nguyen, ${ }^{5}$ Trinh Cong Tran $\mathbb{D}^{\circ},{ }^{6}$ and Chien Ngoc Nguyen $\mathbb{D}^{2,3}$ \\ ${ }^{1}$ Faculty of Pharmacy, University of Medicine and Pharmacy, Hue University, 6 Ngo Quyen, Hue, Thua Thien Hue, Vietnam \\ ${ }^{2}$ National Institute of Pharmaceutical Technology, Hanoi University of Pharmacy, 15 Le Thanh Tong, Hanoi, Vietnam \\ ${ }^{3}$ Department of Pharmaceutical Industry, Hanoi University of Pharmacy, 15 Le Thanh Tong Hanoi, Vietnam \\ ${ }^{4}$ Department of Organic Chemistry, Hanoi University of Pharmacy, 15 Le Thanh Tong, Hanoi, Vietnam \\ ${ }^{5}$ Department of Pharmaceutics, Hanoi University of Pharmacy, 15 Le Thanh Tong, Hanoi, Vietnam \\ ${ }^{6}$ Department of Biology and Microbiology, Hanoi University of Pharmacy, 15 Le Thanh Tong, Hanoi, Vietnam
}

Correspondence should be addressed to Trinh Cong Tran; congdhn@gmail.com and Chien Ngoc Nguyen; nguyenngocchien@yahoo.com

Received 9 September 2020; Revised 6 November 2020; Accepted 14 December 2020; Published 31 December 2020

Academic Editor: Hassan Azzazy

Copyright ( 2020 Hoang Nhan Ho et al. This is an open access article distributed under the Creative Commons Attribution License, which permits unrestricted use, distribution, and reproduction in any medium, provided the original work is properly cited.

\begin{abstract}
Fungal infection of the skin is one of the most common dermatological diseases in the world. Gel formulations are among the most suitable dosage forms for topical use to treat cutaneous infection. Nanotechnology is a promising approach to penetrate the deeper skin layers and enhance permeability of itraconazole (ITZ) through the stratum corneum. ITZ-loaded nanoparticles (ITZ NPs) were fabricated using the evaporation emulsion method, followed by incorporation of NPs into gel using Carbopol 934 as the gelforming excipient. The physical properties, in vitro release, ex vivo permeation studies, and antifungal activity of ITZ NP gel were characterized. ITZ NPs were almost spherical in shape with colloidal sizes in the range of $200 \mathrm{~nm}$. The drug encapsulation efficiency was $98.79 \pm 1.24 \%$. ITZ NP gel demonstrated a sustained ex vivo permeation of ITZ over $24 \mathrm{~h}$ through excised rat skin and a higher drug penetrating capacity than that of a gel containing ITZ-saturated suspension. The in vitro antifungal activity of the ITZ-loaded NP incorporated gel was better than that of ITZ dispersion. Incorporation of the ITZ-loaded nanosystem into gel has the potential to enhance antifungal activity through transdermal drug delivery.
\end{abstract}

\section{Introduction}

Itraconazole (ITZ), an antifungal agent of the triazole group, has a broad activity spectrum. ITZ is popularly used for the treatment of fungal infections due to its good tolerance for patients. ITZ is a drug of choice for immunocompromised and nonimmunocompromised patients along with patients who cannot tolerate amphotericin B therapy [1]. However, its hydrophobic properties and a large variation in oral bioavailability between individuals have limited its use. Moreover, its adverse effects have been reported such as constipation, abdominal pain, headache, and in rare instances, cardiac failure. Its other disadvantage is it not being recommended for patients with renal and/or hepatic impairment $[2,3]$.
Topical drug delivery is the conventional administration route for the treatment of cutaneous disorders such as acne, aging, or some cutaneous diseases such as skin inflammation. Gel, a semisolid form, is generally prepared by swelling a cross-linked polymer network in a liquid medium. The interaction between the solid-state polymer and the liquid component decides its properties. In comparison to creams and ointments, gel formulations for topical use are suitable for delivering drugs owing to less greasiness and easy removal from the skin.

The drug delivery onto the skin is found effective for local therapy in the treatment of many dermatological diseases such as fungal infection. However, the main challenge facing the efficiency of topical antifungal drugs is the ability to penetrate the required layers of the skin through the stratum 
corneum, the first layer of the skin, for a highly effective and successful treatment $[4,5]$. Additionally, adequate drug concentrations in skin layers play an important role in ensuring the effective therapy via topical administration. Several attempts have been made to overcome these shortcomings and to discover and develop appropriate dosage forms which can enhance effectiveness and patient compliance while maintaining safety, efficacy, and affordability of drug delivery [5]. Among them, nanostructured drug carriers have been the alternative approaches in formulations in the past decade for topical administration [6]. Furthermore, nanocarriers could enhance drug penetration through the skin, and thus, help eradicate deep fungal infections. Other attractive features of nanoscale particles include sustained drug release, enhanced drug stability, targeting of infected tissue, reduction of off-target side effects, and improved drug efficacy [7].

Nanosuspensions, one of the nanotechnological products, have been growing in popularity with much application in various drug administration routes. However, the low viscosity of nanosuspensions poses the problem of applicability to skin and decreases patient compliance. Nevertheless, compared with conventional solution, gel, or cream formulations, nanosuspension-incorporated gels significantly enhance the absorption of drugs. Consequently, the combination of nanosuspension with bioadhesive gel may be a promising approach to treat topical infection. The nanosuspensionbased gel may overcome the gastric side effects of ITZ and be retained on skin with improved therapeutic outcome for an extended period of time [3].

Polymers derived from cellulose derivatives are often used to formulate the drug delivery systems with specific purposes such as enteric coating, colon targeting, controlled release, and taste masking. Ethylcellulose (EC), one of the water-insoluble cellulose derivatives, is often used for the preparation of the abovementioned drug delivery systems [8]. Quercetin-loaded EC nanoparticles (NPs) were reported to control the drug release and increase drug skin retention [9]. Vitamin C-loaded EC NP-incorporated hydroxypropyl methylcellulose gel used for topical delivery has been reported to extend drug release, significantly improve therapeutic effect, and considerably reduce adverse reactions [10]. Dexamethasone-loaded EC NPs controlled drug release on the skin surface; hence, this formulation was promising in the treatment of some skin diseases [11].

Therefore, the present study is aimed at fabricating ITZloaded NP-incorporated gels (ITZ NP gel) for topical administration and to evaluate their in vitro antifungal activities. The physicochemical characterization of these formulations was conducted, such as the particle size, polydispersity index (PDI), morphology, and physical state. The subsequent in vitro or ex vivo studies of these formulations were performed to investigate their skin permeation and antifungal efficacy.

\section{Materials and Methods}

2.1. Materials. All chemicals were used without further purification and obtained from the following commercial sources: ITZ (Yashica Pharmaceuticals Private Limited,
Maharashtra, India), EC (Shanghai Colorcon Coating Technology Ltd., Shanghai, China), dichloromethane (Xilong Scientific Co., Ltd., Guangdong, China), polyvinyl alcohol (Kuraray Asia Pacific Pte. Ltd., Singapore), polysorbate 80 (Tween 80; Duksan Chemical Co., Ansan, South Korea), propylene glycol (PG, Zhejiang Kehong Chemical Co., Zhejiang, China), and Carbopol 934 (CBP 934; Lubrizol Corp., Wickliffe, Ohio, USA). Methanol was of high-performance liquid chromatography (HPLC) grade. Other chemicals were of analytical grade.

Candida albicans ATCC 10231 and Aspergillus niger ATCC 16404 (National Institute of Drug Quality Control, Vietnam) used for the antifungal activity test were a gift from the Department of Industrial Pharmacy, Hanoi University of Pharmacy. The rat skin was a gift from the National Institute of Drug Quality Control (Vietnam).

2.2. Preparation of ITZ NPs. ITZ NPs were prepared using the solvent evaporation method [12]. In brief, ITZ and EC were dissolved in $5 \mathrm{~mL}$ dichloromethane. The oil phase was added slowly to $10 \mathrm{~mL}$ of the aqueous phase containing $2 \%$ polyvinyl alcohol $(w / v)$ with a flow rate of $2 \mathrm{~mL}$ per min and was homogenized by probe sonication at an input power of $117 \mathrm{~W}$ (Ultrasonic processor Vibra-Cell, Sonics \& Materials, USA) and magnetic stirring at $1000 \mathrm{rpm}$ (Ika RCT basic IKAMAG, Germany) for $5 \mathrm{~min}$ in a cold ice water bath (5$\left.10^{\circ} \mathrm{C}\right)$. Then, the resultant emulsion was magnetically stirred at $1000 \mathrm{rpm}$ for $3 \mathrm{~h}$ to evaporate dichloromethane to obtain the ITZ suspension. The emulsifier and free drug were washed three times by a Millipore UFC801008 Amicon ${ }^{\circledR}$ filter (Amicon Ultra, Millipore, USA) with a molecular weight cut-off of $10 \mathrm{kDa}$.

Lyophilization of the ITZ dispersion was performed using a freeze dryer (Christ Alpha 1-LD, Germany). The dispersion was prefrozen $\left(-70^{\circ} \mathrm{C}\right)$ for $12 \mathrm{~h}$ and subsequently lyophilized at $-48 \pm 2^{\circ} \mathrm{C}$ and $0.120 \pm 0.020 \mathrm{mbar}$ for $24 \mathrm{~h}$.

2.3. Preparation of Gel Formulations. ITZ NPs were introduced into hydrogel using CBP 934 as the gel-forming excipient and $P G$ as the permeability enhancer. Batches of hydrogel formulations ( $10 \mathrm{~g}$ ) were fabricated by adding ITZ nanosuspensions to an aqueous dispersion containing CBP 934 and $5 \%$ of PG ( $w / v$, of the total volume). Hydrogels were gently stirred overnight and then stored at $4^{\circ} \mathrm{C}$ until use [13]. Gel containing ITZ-saturated suspension was prepared by magnetic stirring of ITZ $(0.1 \mathrm{~g})$ in $10 \mathrm{~mL}$ of water overnight for $72 \mathrm{~h}$, followed by mixing with the aqueous dispersion containing CBP 934 and PG, and then, stirring overnight to form ITZ-saturated hydrogel as described in the preparation process of the ITZ NP gel.

\subsection{Characterization of ITZ NPs}

2.4.1. Determination of Particle Size (Z) and PDI. The ITZ NPs were characterized by measuring $Z$ and PDI using a dynamic light-scattering system, Zetasizer Nano 90 (Malvern Instruments, UK). The samples were diluted 20 times with distilled water prior to $Z$ and PDI analyses (triplicate measurements within each sample). 
2.4.2. Morphological Characterization. The morphology of ITZ NPs and ITZ NP gel was evaluated using a highresolution scanning electron microscope (SEM; Hitachi S4800, Tokyo, Japan). For SEM, the samples were dropped on the aluminum foil and dried at room temperature $\left(25 \pm 2^{\circ} \mathrm{C}\right)$. The aluminum foil was mounted on aluminum/copper stubs with carbon tapes and without magnetron and sputter-coated by a $3 \mathrm{~nm}$ gold layer before SEM imaging as described in our previous paper [14].

2.4.3. X-Ray Powder Diffraction (XRPD) Analysis. The XRPD analysis was performed on ITZ, EC, physical mixture, and lyophilized NPs using a D8 ADVANCE diffractometer (Bruker, Germany) with $\mathrm{Cu} \mathrm{K} \alpha$ radiation $(\lambda=1.5406)$ between $5^{\circ}$ and $50^{\circ}(2 \theta)$ at room temperature $\left(25 \pm 2^{\circ} \mathrm{C}\right)$ with a step size of $0.02^{\circ}$. The total measuring time was $498 \mathrm{~s} / \mathrm{step}$. The operating current and voltage were $40 \mathrm{~mA}$ and $40 \mathrm{kV}$, respectively [14].

2.4.4. Fourier Transform Infrared Spectroscopy (FT-IR) Analysis. The surface chemistry and physical interaction between the polymers and drug were determined from FTIR spectra. The samples for FT-IR spectrometry were mixed with $\mathrm{KBr}$ and compressed into transparent tablets prior to the analysis over the $4000-400 \mathrm{~cm}^{-1}$ range using a Jasco 6700 FT-IR spectrometer (Japan).

2.4.5. Encapsulation Efficiency and Drug Loading. The values of encapsulation efficiency and drug loading capacity of ITZ NPs were determined using HPLC. The ITZ nanosuspension $(2 \mathrm{~mL})$ was loaded into a Millipore UFC801008 Amicon ${ }^{\circledR}$ filter (Amicon Ultra, Millipore, USA) with a molecular weight cut-off of $10 \mathrm{kDa}$ and centrifuged at $3000 \times g$ for 30 min (Hermle, Germany). Unbound ITZ, which moved across the filter membrane to the bottom compartment, was assayed, and the drug encapsulation efficiency (EE) and the drug loading capacity (LC) were then calculated using the following equation $[13,15]$ :

$$
\begin{aligned}
& \mathrm{EE}(\%)=\frac{\left(W_{\text {initial drug }}-W_{\text {unbound drug }}\right)}{W_{\text {initial drug }}} \times 100, \\
& \mathrm{LC}(\%)=\frac{\left(W_{\text {initial drug }}-W_{\text {unbound drug }}\right)}{W_{\text {nanoparticles }}} \times 100,
\end{aligned}
$$

where $W$ represents the weight in $\mathrm{mg}$.

2.4.6. HPLC Assay. ITZ was assayed chromatographically using an Agilent HPLC system and a C18 analytical column (Zorbax Eclipse XBD C18; $250 \times 4.6 \mathrm{~mm}, 5 \mu \mathrm{m}$; Agilent, USA) at ambient temperature. The mobile phase comprised methanol and purified water $(80: 20, v / v)$. The flow rate was $1.5 \mathrm{~mL} / \mathrm{min}$, the injection volume was $20 \mu \mathrm{L}$, and the effluent was monitored at $265 \mathrm{~nm}$ [16].

\subsection{Characterization of ITZ NP Gel}

2.5.1. Visual Appearance and $p H$. Visual appearance and clarity of the prepared ITZ NP gel were observed for the presence of any particulate matter and uniformity. The $\mathrm{pH}$ of each gel batch was determined using a $\mathrm{pH}$ meter (Mettler Toledo, Switzerland). $\mathrm{pH}$ was measured by dispersing $1 \mathrm{~g}$ of each formulated gel in $10 \mathrm{~mL}$ of distilled water [4].

2.5.2. In Vitro Release Study. In vitro drug release was determined over a period of $24 \mathrm{~h}$ using a cellulose acetate synthetic membrane with a molecular weight cut-off of $12 \mathrm{kDa}$ (SigmaAldrich, Missouri, USA). The studies were conducted using static Franz diffusion cells (Hanson Research, CA, USA) with a diffusion area of $1.767 \mathrm{~cm}^{2}$ and a receptor compartment containing $7 \mathrm{~mL}$ phosphate-buffered saline at pH 7.4 [17, 18]. This receptor phase was stirred at $400 \mathrm{rpm}$ and maintained at $37 \pm 0.5^{\circ} \mathrm{C}$ via a thermostatic water pump. Each sample was applied with an equivalent amount of $2 \mathrm{mg}$ drug, and at predetermined points of time, aliquots of $1 \mathrm{~mL}$ were removed and replaced with the fresh medium. All ITZ concentrations were assayed using the HPLC method described in Section 2.4.6.

2.5.3. Rheological Analysis. The rheological analysis of the CBP 934 gel with the introduced ITZ NP (ITZ NP gel) was performed using a controlled stress rheometer (Discovery Hybrid Rheometer HR-1; TA Instruments, USA), equipped with a stainless steel plate (diameter of $40 \mathrm{~mm}$ and cone angle of $4^{\circ}$ ). Briefly, ITZ NP gel ( $1.2 \mathrm{~g}$ ) was allowed to stand on the plate for $60 \mathrm{~s}$ to eliminate the external force caused by putting the sample on the plate, and the sample was maintained at a homogeneous temperature. Next, the viscosity and the corresponding shear stress of the ITZ NP gel were determined isothermally at $37^{\circ} \mathrm{C}$ with a shear rate working range of $0-900 \mathrm{~s}^{-1}$. The elastic $\left(G^{\prime}\right)$ and loss $\left(G^{\prime \prime}\right)$ moduli were recorded [19].

2.6. Ex Vivo Permeation Study. Ex vivo permeation studies were also performed in static Franz diffusion cells (as described in Section 2.5.2) using the male rat epidermis as a skin model; this was clamped between the donor and receptor compartments with the stratum corneum side facing up [20]. The rat skin was treated as described previously with permission obtained from the Ethics Commitment Jury [21]. The fur and lipids were removed from the skin. Intact skin specimens were rinsed in $0.9 \%$ saline and covered with aluminum foil before storage at $-20^{\circ} \mathrm{C}$. Before the experiments, the skin was warmed to room temperature $(25 \pm 2$ $\left.{ }^{\circ} \mathrm{C}\right)$. The lower face of the skin was then immersed in a $0.9 \%$ saline solution or release media for $30 \mathrm{~min}$.

The retained drug in the skin was assessed by measuring the drug amount in the skin after permeability studies. The drug was extracted into methanol by means of sonication and centrifugation. Specifically, the skin was rinsed thrice with saline buffer ( $\mathrm{pH} 7.4$ ) and then broken into small pieces and placed in a $10 \mathrm{~mL}$ volumetric flask. After adding $5 \mathrm{~mL}$ methanol, the samples were sonicated thrice for $30 \mathrm{~min}$ each. The collected solutions were centrifuged at $8000 \mathrm{rpm}$ for $10 \mathrm{~min}$. The supernatants were then filtrated through a $0.45 \mu \mathrm{m}$ membrane before quantification via HPLC [13].

2.7. Antifungal Activity. The procedure for evaluating antifungal activity was the same as the potato-dextrose agar method with some modifications [22]. Sabouraud agar was dissolved in distilled water to a final concentration of $70 \mathrm{~g} / \mathrm{L}$ 


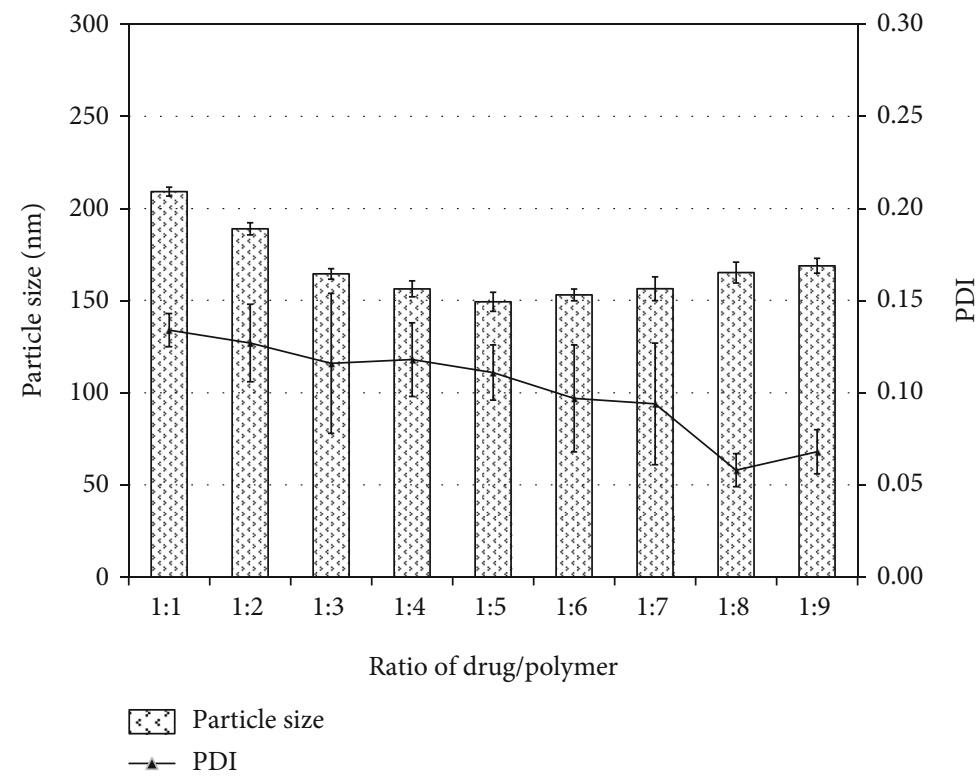

FIgURE 1: The size and PDI of ITZ NPs at various ratios of drug/polymer.

(peptone: $10 \mathrm{~g}$, glucose: $40 \mathrm{~g}$, agar: $20 \mathrm{~g}$ ) and then sterilized at $121^{\circ} \mathrm{C}$ for $15 \mathrm{~min}$. The sterilized Sabouraud agar solution was placed in a water bath, and the temperature was cooled and maintained at 55 to $60^{\circ} \mathrm{C}$. The antifungal agent stock solutions (500-8000 ppm, dissolved in dimethyl sulfoxide) or ITZ NP gel was mixed with the Sabouraud agar solution was used to produce a series of different final concentrations. Drug-free agar containing only $0.5 \%(v / v)$ dimethyl sulfoxide and blank NP gel were used as controls. The mixtures of antifungal agents and Sabouraud agar solutions were poured directly into the wells $(1.0 \mathrm{~mL} /$ well in 12 -well plates). After the plates were cooled to room temperature $\left(25 \pm 2^{\circ} \mathrm{C}\right)$, $10 \mu \mathrm{L}$ of freshly prepared fungal suspension $\left(2 \times 10^{3} \mathrm{cfu} / \mathrm{mL}\right)$ was inoculated onto the agar of each well. The plates were incubated aerobically at $35^{\circ} \mathrm{C}$ for $36-48 \mathrm{~h}$ until the fungi of the control cultures (containing no drug) reached a growth sufficient for identification. A four-grade scoring system was used (i.e., the control cultures were scored as 4; no detectable fungal growth was scored as 0 ; and 3, 2, and 1 represented 75,50 , and $25 \%$ the fungal growth of the control cultures, respectively).

2.8. Data Analysis. The release experiments were performed in triplicate; the results are expressed as the mean \pm SD. Differences between groups were analyzed using ANOVA test. $p<0.05$ was considered statistically significant.

\section{Results and Discussion}

3.1. Preparation of ITZ NPs. Using the emulsion solvent evaporation technique, two critical parameters, the drug/polymer ratio and drug content, were assessed to achieve optimal conditions for the preparation of a nanoscale drug delivery system. Polymers can form a thin layer covering drug particles, which contributes to increasing the stability of the nanosuspension.
Figure 1 shows a narrow size distribution range of below $300 \mathrm{~nm}$ for the polymer-based formulations. When EC concentration was increased, the average particle size of EC NPs was in the range of 150 to $200 \mathrm{~nm}$ with PDI value less than 0.15 for the formulations containing drug : polymer ratios of $1: 5$ to $1: 1$, respectively. When the polymer concentration is low, it could be insufficient to cover the surface of all particles, and uncoated particles tend to accumulate into bigger particles. However, when the polymer content was further increased, the particle conversely exhibited a larger size. This could be attributed to the high viscosity of the oil phase, and a poorer dispersibility of the oil phase in the aqueous phase. Moreover, the high viscosity of the solution enhances the aggregation in a more concentrated solution, thereby forming bigger emulsion droplets [10, 23]. Consequently, the effects of different ITZ/polymer ratios (1:3 to $1: 5)$ on the size and size distribution were further evaluated to find the optimal ratios.

As shown in Figure 2, when the ITZ concentration increased in relation to polymer mass, the particle size and PDI increased. This can be explained by the fact that a greater amount of drug results in a more viscous dispersed phase, making the mutual dispersion of the phases difficult and originating larger particles [23]. The ratio of drug : polymer of $1: 3$ and drug concentration of $1.0 \%$ were selected for further study owing to its small size, narrow size distribution range $(220.7 \pm 3.6$ $\mathrm{nm}$ and $0.095 \pm 0.034$, respectively, Figure 3(a), $p>0.05$ ), encapsulation efficiency approximately $100 \%(98.79 \pm 1.24$ $\%)$, and high loading capacity $(11.11 \pm 0.48 \%)$ compared to other ratios $(9.80 \pm 0.33 \%$ and $8.58 \pm 0.58 \%$ for the ratio of drug : polymer of $1: 4$ and $1: 5$, respectively, $p<0.05)$.

\subsection{Characterization of ITZ NPs}

3.2.1. Morphology. Figure 3(b) shows the representative SEM image of ITZ-loaded NPs. The NPs are spherical in shape and a size range of within $200 \mathrm{~nm}$. 


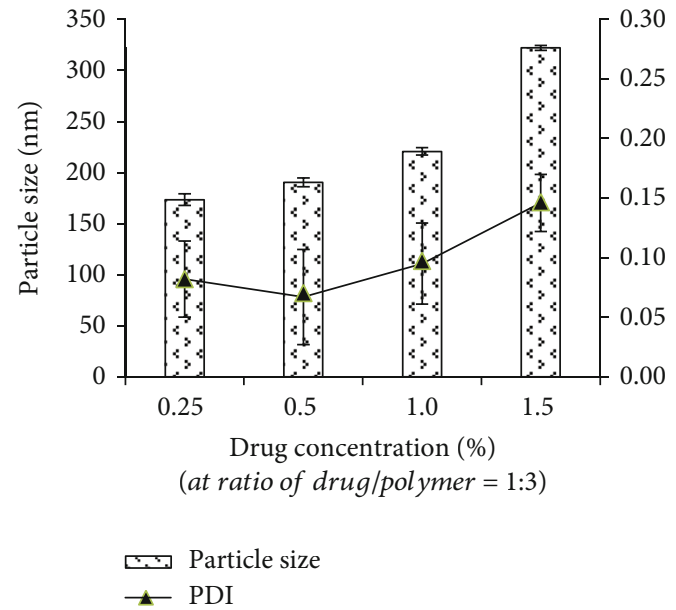

(a)

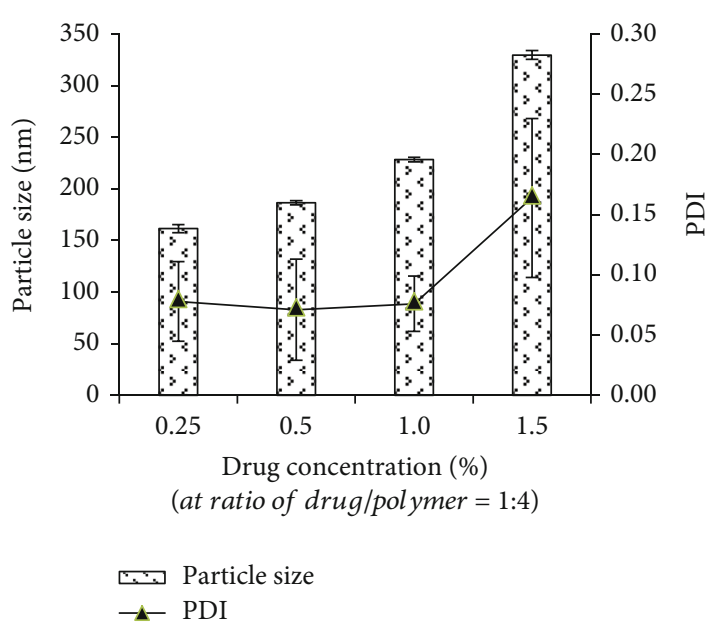

(b)

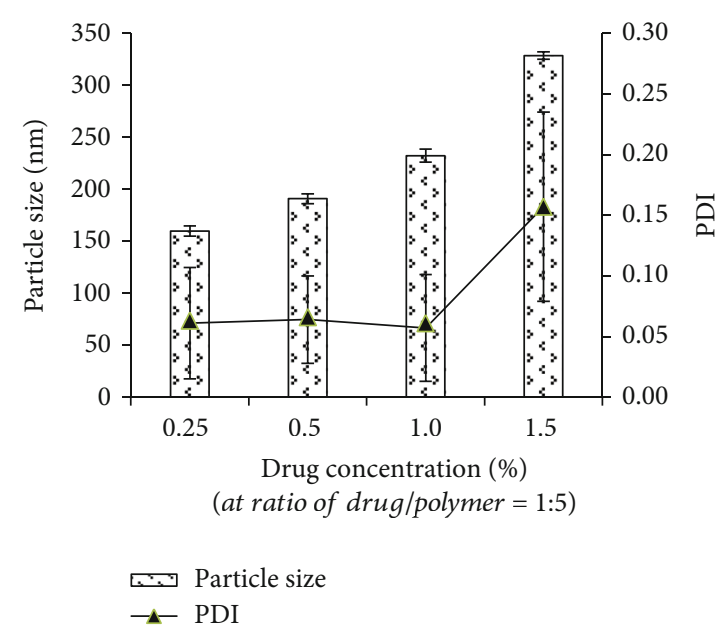

(c)

FIgURE 2: The size and PDI of ITZ NPs at different drug concentrations and drug/polymer ratios: (a) at ratio of drug/polymer $=1: 3$, (b) at ratio of drug/polymer $=1: 4$, and $(\mathrm{c})$ at ratio of drug/polymer $=1: 5$.

3.2.2. XRPD Analysis. The XRPD patterns of pure materials (ITZ, EC), lyophilized ITZ-loaded NPs (NPs), and the respective physical mixture (PM) are shown in Figure 4. The diffraction pattern of ITZ showed numerous distinct reflections, thus indicating the crystalline nature of the drug. The reflections of ITZ were observed in the range of $5^{\circ}-30^{\circ}$ at diffraction angles of $2 \theta$ at $14.6^{\circ}, 17.6^{\circ}, 18.1^{\circ}, 19.5^{\circ}, 20.5^{\circ}, 23.6^{\circ}$, and $25.6^{\circ}$ (Figure 4 , marked with “*”) [24]. The XRPD of EC polymer showed a broad peak at $20.5^{\circ}$, indicating its amorphous state [25]. As shown in Figure 4, the distinctive sharp reflections of ITZ disappeared in the XRPD patterns of NPs, although these were observed in the XRPD patterns of a corresponding PM, indicating that ITZ was in an amorphous phase in NPs.

3.2.3. FT-IR Analysis. FT-IR studies were performed to detect the possible interactions between the ITZ and other components in the ITZ NPs. Figure 5 shows the IR spectra of ITZ, EC, and the NP formulation. Pure ITZ displays a band with a strong peak at $1699 \mathrm{~cm}^{-1}$ indicative of the $\mathrm{C}=\mathrm{O}$ stretch of the ketone group, a sharp peak at $1510 \mathrm{~cm}^{-1}$ attributed to an aromatic C-C stretch band, and a distinctive peak at $1228 \mathrm{~cm}^{-1}$ owing to aromatic C-H in-plane bending. The peaks around $3000 \mathrm{~cm}^{-1}$ were formed owing to stretching vibrations of amino groups [26]. The EC IR spectrum showed a band at $3476 \mathrm{~cm}^{-1}$, which could be attributed to the $\mathrm{OH}$ stretching vibration [27]. The PM spectrum was the sum of the spectrum of ITZ, polyvinyl alcohol, and EC. A shift in the $\mathrm{C}=\mathrm{O}$ band of ITZ in the NP spectrum from 1699 to $1719 \mathrm{~cm}^{-1}$ may be attributed to the change in the solid-state form of ITZ from crystalline to amorphous during the preparation of NPs. The relative similarity of the other areas of the spectra of NPs and PM suggests that there was no chemical change between the drug and the other components.

3.3. Preparation and Characterization of ITZ NP Gel. To overcome the disadvantages of polymeric NPs such as low viscosity and low bioadhesive ability, the gel formulation was employed for topical administration. Therefore, the influence of the gel-forming polymer CBP 934 (triethylamine was used to adjust to $\mathrm{pH} 6-7,5 \%(w / v)$ of PG) on gel properties was investigated. 


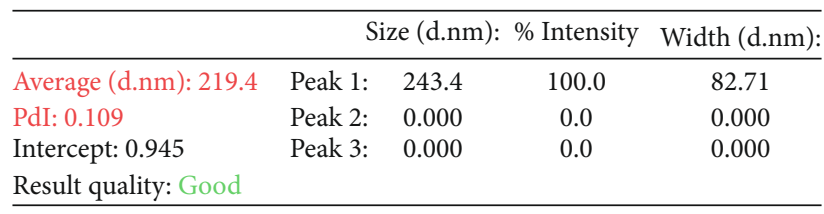

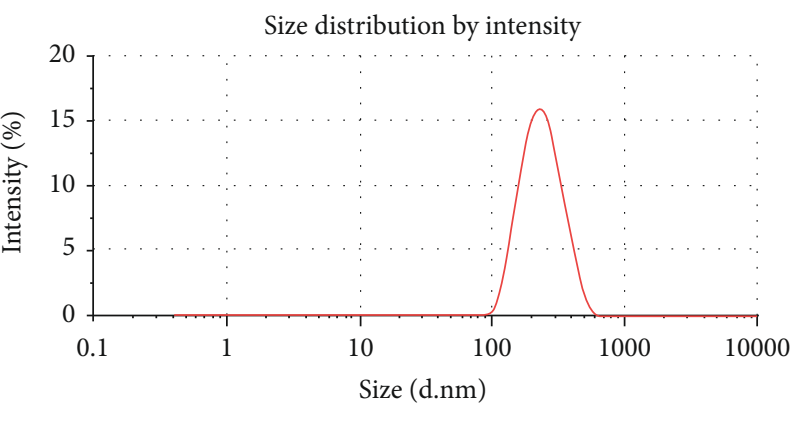

Record 1740: EC 1

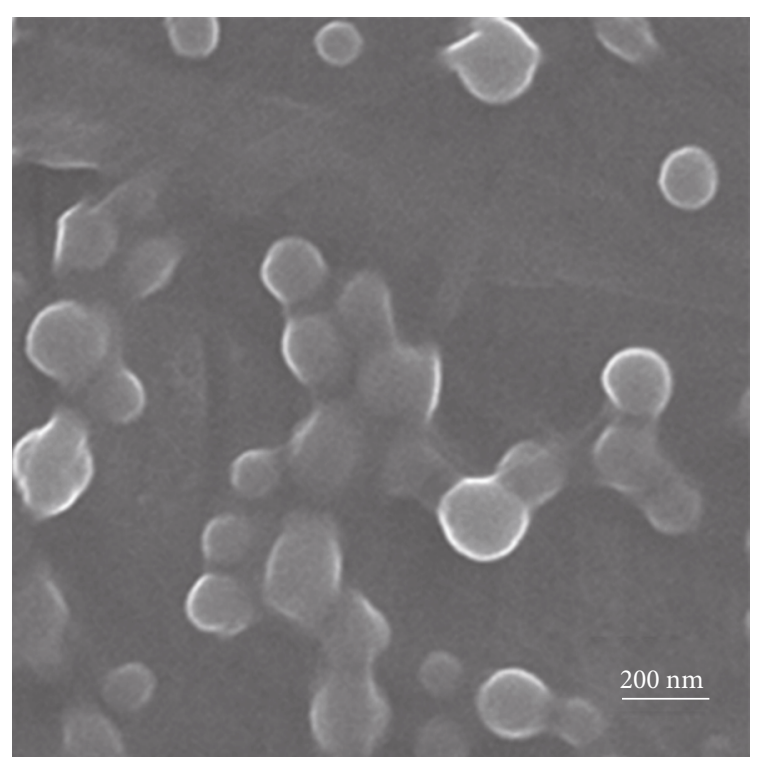

(b)

FIgure 3: (a) DLS result and (b) SEM image of ITZ NPs (scale bar $=200 \mathrm{~nm}$ ).

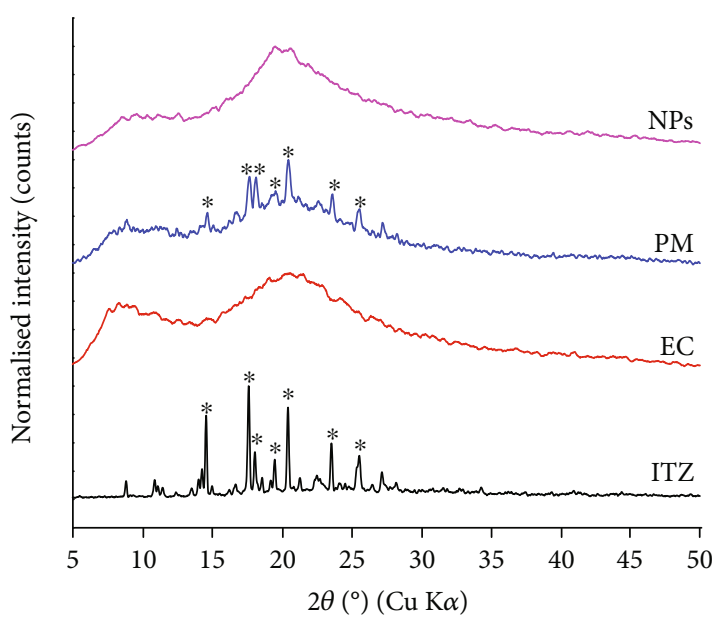

FIGURE 4: X-ray powder diffraction patterns (XRPD) of pure materials (ITZ, EC), NPs, and the respective PM (PM). Characteristic reflections of ITZ is marked with $\left({ }^{*}\right)$. All spectra are normalized.

3.3.1. Visual Appearance and $p H$. Table 1 shows the physicochemical properties of several CBP gel formulations such as visual appearance, $\mathrm{pH}$ of gel containing saturated suspension, and ITZ NP gel.

3.3.2. In Vitro Drug Release Study. Figure 6(a) shows the release profiles of ITZ from gel containing saturated suspension and ITZ NP gel. The NP gel exhibited an initial burst release followed by a sustained release for $24 \mathrm{~h}$. The burst release could be explained by the presence of the drug adsorbed onto or near the nanoparticle surface [10]. Compared to gel containing ITZ-saturated suspension, nanosystem application led to the formation of a gel with better performance and with a sustained release pattern. The amount of the drug released at $24 \mathrm{~h}$ was $0.12 \pm 0.02 \%$ and
$16.92 \pm 0.50 \%$ for gel containing ITZ-saturated suspension and ITZ NP gel, respectively. In this study, EC, a releasecontrolling excipient with hydrophobic but water permeable character influencing the controlled drug release from the modified drug delivery system, was used as the encapsulating polymer [10]. This result was consistent with that reported in previous studies on EC NP incorporated into hydroxypropyl methylcellulose gel [10] or on lipid NP incorporated into CBP gel [28].

The concentration of gel-forming excipient could adjust the viscosity and bioadhesive properties of gel. Hence, in this study, CBP 934 concentration from $0.3 \%$ to $0.5 \%(w / v)$ was investigated.

As shown in Figure 6(b), the concentration of gelforming excipient exhibited an influence on drug release, e.g., at the concentration of $0.3 \%$ CBP 934, maximum drug content was released $(29.85 \pm 0.70 \%$ after $24 \mathrm{~h})$. With the increase of CBP 934 concentration from $0.3 \%$ to $0.5 \%$, drug release was reduced (from $29.85 \pm 0.70 \%$ to $16.92 \pm 0.50 \%$ after $24 \mathrm{~h}, p<0.05)$. CBP 934 may influence the viscosity of a gel in a concentration-dependent manner, which has been reported to have an effect on the dissolution rate. The higher the polymer concentration, the greater is gel viscosity, which results in a reduction in drug release [13]. Consequently, $0.3 \%$ CBP 934 was finally chosen owing to its high dissolution rate and good bioadhesive properties.

3.3.3. Rheological Analysis. In oscillatory rheometry, the effects of oscillatory stress on the viscoelastic properties are measured as the storage modulus $\left(G^{\prime}\right)$, a measure of the elasticity, and the loss modulus $\left(G^{\prime \prime}\right)$, representing viscous components at a given frequency of oscillation. An ideal gel formulation should exhibit a solid-like mechanical spectrum, where $G^{\prime}>G^{\prime \prime}$ with little frequency dependence [29]. Based on the results of rheological studies, the ITZ gel prepared 


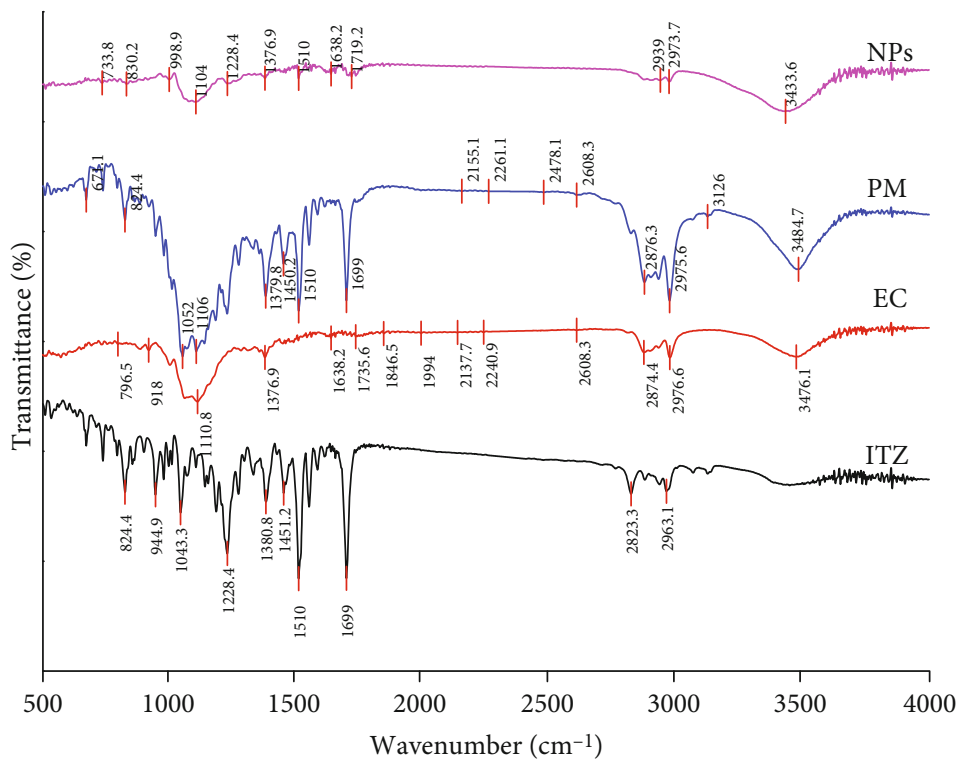

Figure 5: FT-IR spectra of pure materials (ITZ, EC), NPs, and the respective PM (PM) (1510-1451 (armomatic C-C stretches); 1228.4-1043.3 (aromatic C-H in plane bends); 1228 alkyl-aryl ether, $1699 \mathrm{C}=\mathrm{O}$; heterocyclic amine, $\mathrm{NH}$ stretch: 3490-3430; and aromatic tertiary amine, $\mathrm{CN}$ stretch: 1360-1310).

TABLE 1: Visual appearance and $\mathrm{pH}$ of $\mathrm{CBP}$ gel formulations.

\begin{tabular}{l}
$\begin{array}{l}\text { Delivery system } \\
\text { (equivalent to } 10 \mathrm{~mL})\end{array}$ \\
\hline CBP $934(\%, w / v)$
\end{tabular}

CBP: Carbopol 934; ITZ: itraconazole.

with $0.3 \%$ CBP was observed to be frequency independent and have a typical solid-like mechanical spectrum $\left(G^{\prime}>G^{\prime \prime}\right)$ (Figure 7(a)).

Moreover, the rheological behavior of the prepared gels was investigated because it plays an important role in their flow characteristics during the preparation process, packaging, storage, and application to the skin [30]. In our study, the changes in shear stress versus shear rates were used to determine whether the flow properties of the formulations were Newtonian [29]. As shown in its flow curve (Figure 7(b)), ITZ NP gel formulation was determined to have a non-Newtonian plastic behavior. As a typical behavior of non-Newtonian plastic fluids, this system also started flowing upon reaching a certain yield value. In addition, the prepared ITZ NP gel exhibited no thixotropy because there were no hysteresis loops in the rheogram, reflecting no time-dependent decrease in viscosity under stress and stable structure of the polymer used in the preparation [30]. 




(a)

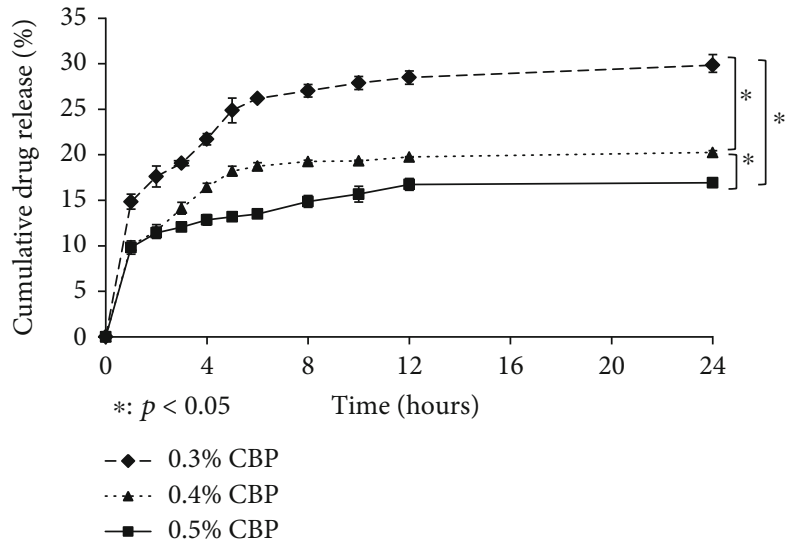

(b)

FIGURE 6: In vitro drug release profiles (a) of ITZ NP gel and gel containing saturated suspension $(n=3)$ and (b) of ITZ NP gel with different CBP concentrations $(n=3)$.

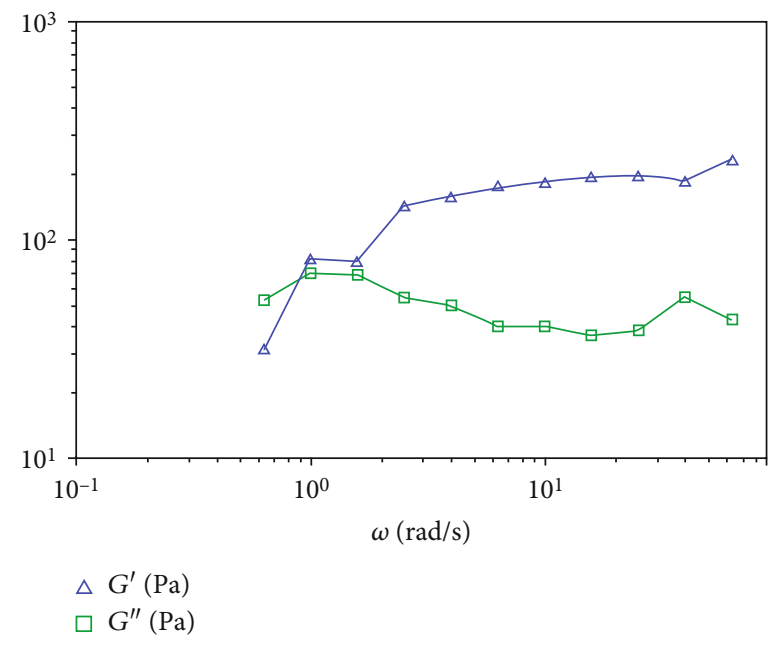

(a)

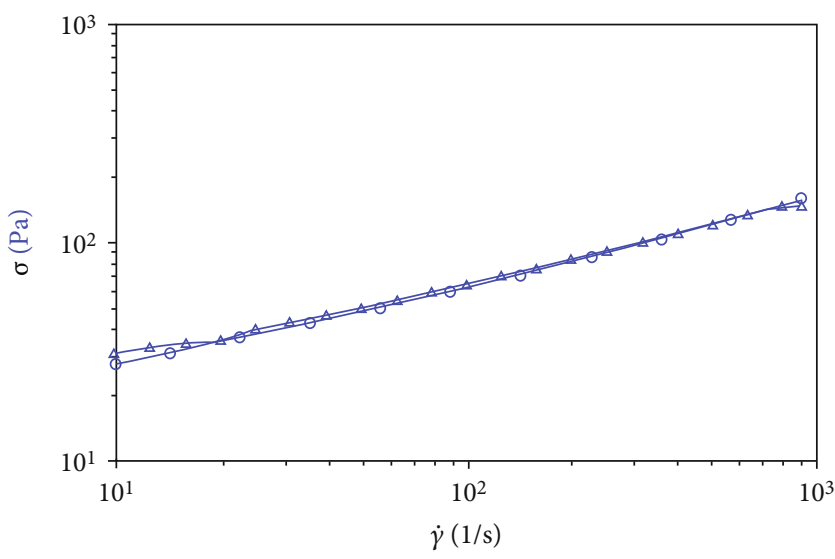

(b)

FIGURE 7: (a) Frequency-dependent changes in viscoelastic properties $\left(G^{\prime}\right.$, storage modulus, the ITZ NP gel formulation.

3.4. Ex Vivo Skin Permeation Study. The gel formulations were characterized ex vivo for skin permeation profiles. The retained drug in the skin was then assessed by measuring the drug amount in the skin after $24 \mathrm{~h}$ permeability studies.

As shown in Figure 8, the ITZ NP gel exhibited a higher permeability through mouse skin than gel containing ITZsaturated suspension at all time points. The permeability profile of the ITZ NP gel showed that the amounts of permeated drug were low at the initial time points. Subsequently, the drug permeability was increased and in an extended-release manner. At $24 \mathrm{~h}$, the cumulative amount of drug from ITZ NP gel was found to be $353.25 \pm 17.56\left(\mu \mathrm{g} / \mathrm{cm}^{2}\right), 20$-fold higher than that from gel containing ITZ-saturated suspension. It has been reported that an interval of time is required for drugs released from nanocarriers as well as a gel matrix to reach the skin and penetrate the skin into the release media. The nanosized system could improve drug delivery efficacy by enhancing drug penetration to the entire depth of the skin $[13,31]$. In addition, the surfactant also swells the stratum

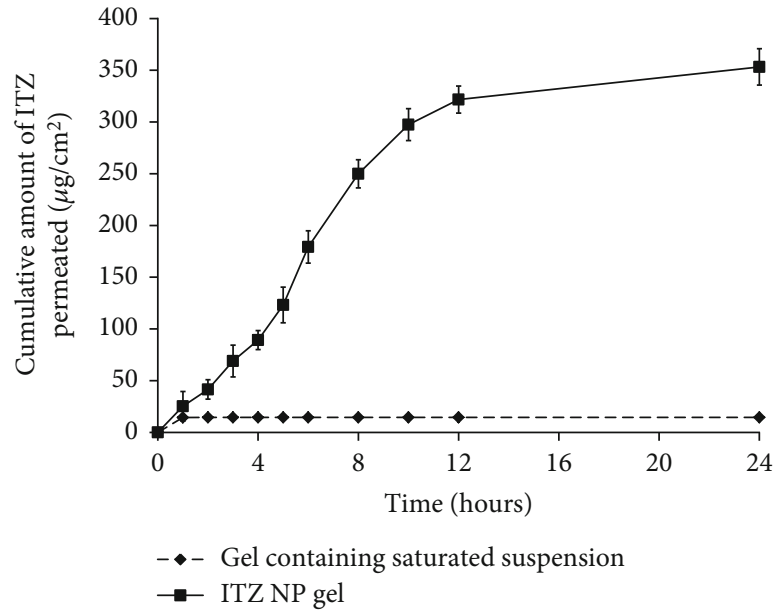

FIgUre 8: Permeation profiles of ITZ NP gel and gel containing ITZ-saturated suspension $(n=3)$. 


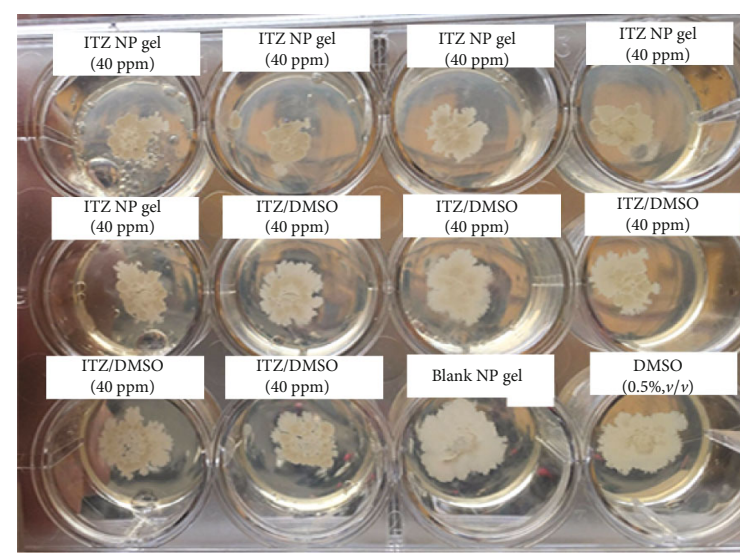

(a)

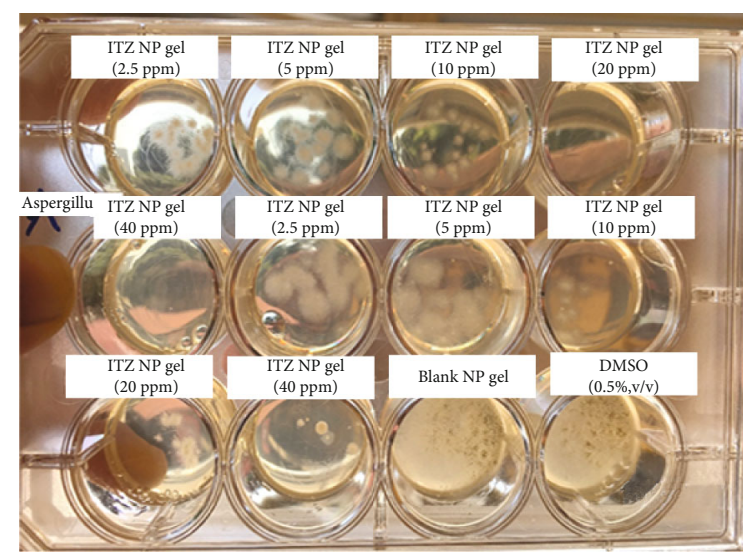

(b)

FIgURE 9: Antifungal activity of ITZ NP gel and ITZ dispersion on (a) Candida albicans and (b) Aspergillus niger.

corneum and the intact vesicle can penetrate through the intact skin cells [32].

Moreover, the amount of retained drug of the ITZ NP gel in the skin after $24 \mathrm{~h}$ was also higher than that of gel containing ITZ-saturated suspension $(573.88 \pm 43.35$ $\mu \mathrm{g} / \mathrm{cm}^{2}$ and $79.74 \pm 23.45 \mu \mathrm{g} / \mathrm{cm}^{2}$, respectively, $p<0.05$ ). This could be explained by the effect of covering the skin surface with NPs, NPs inside the cell space, and the better bioadhesive ability of gel. Hence, the ITZ NP gel showed better permeation and drug retention than gel containing ITZ-saturated suspension.

3.5. Antifungal Activity. Candida albicans forms creamy-grey colonies filamenting along their outer border. The filamentous border becomes visible initially, following $24 \mathrm{~h}$ incubation, but is prominent following $48 \mathrm{~h} \mathrm{[33].} \mathrm{As} \mathrm{shown} \mathrm{in}$ Figure 9(a), after $48 \mathrm{~h}$ incubation, the filamentous border was easily seen at all wells. Meanwhile, Aspergillus species, which also form filaments or hyphae, are the second most common cause of opportunistic fungal infection in humans after Candida albicans. Primary cutaneous infection, especially in immunocompetent patients, is extremely rare, but an increase in prevalence has been noted in the last 20 years [34]. As shown in Figure 9(a), at the concentration of ITZ of $40 \mathrm{ppm}$, the ITZ NP gel exhibited better antifungal activity against $C$. albicans than ITZ dispersion (with a total of 5 points and 10 points, respectively). Figure 9(b) shows that the ITZ NP gel exhibited better antifungal activity against A. niger than ITZ dispersion at all concentrations of ITZ (with a total of 7 points and 10 points, respectively). Furthermore, the blank samples did not show any inhibition on the two fungal species. Nanosized liposomal formulations do not negatively affect the ITZ activity and ITZ preserves its activity even when it is being loaded in liposomes [31]. According to Alhowyan et al. [26], the better inhibition of ITZ NPs (at the concentration of $5 \mu \mathrm{g} / \mathrm{mL}$ ) could be attributed to the capability of NPs to infiltrate across the surrounding media and fungal spores. In addition, the sustained release of ITZ explains the good inhibition over time with the ITZ NP gel as compared to that of ITZ aqueous suspension. Another reason might be that the smaller size of
NPs and their uniform dispersibility increased the contact duration of the NPs with the spores and cells, hence, leading to rapid drug internalization [26]. The study by Ling et al. [15] also showed that the nanoparticle formulation of monomethoxypoly(ethyleneglycol)-b-poly(lactic acid)- (mPEG-bPLA-) based ITZ-loaded NPs preserved the strong inhibition of fungal growth as compared to the ITZ solid dispersion system on C. albicans (strain ATCC 10231) at low ITZ levels (as low as at $0.0153 \mu \mathrm{g} / \mathrm{mL}$ ). Similarly, ITZ-loaded nanostructured lipid carriers protected approximately $100 \%$ of C. albicans fungal infection at $20-40 \mathrm{mg} / \mathrm{kg}$; in contrast, ITZ dispersion (with dimethyl sulfoxide) at either dose did not preserve the health status and life of Galleria mellonella larvae [35]. For the inhibition against Aspergillus species, Patel et al. [36] showed that ITZ-loaded poly(lactic-co-glycolic) acid NPs exhibited enhanced antifungal activity against $A$. flavus compared to ITZ emulsion and ITZ in water at $0.03 \mathrm{mg} / \mathrm{mL}$ ITZ. Consequently, the above findings suggested that the ITZ NP gel could be promising for the development of an effective therapy for cutaneous infection treatment.

\section{Conclusion}

ITZ NPs were successfully fabricated with nanoscale particle size by selecting the appropriate ratios of drug/polymer and drug concentration. ITZ NP gel with a high loading capacity and small particle size ensured a sustained release profile, a good permeation through mouse skin, and a high retained drug content in skin. In addition, compared to the conventional gel, ITZ NP gel exhibited the better in vitro antifungal activity. Furthermore, the optimized gel formulation has the potential to deliver ITZ via topical administration through skin.

\section{Abbreviations}

ITZ: Itraconazole

EC: Ethylcellulose

NPs: Nanoparticles

PDI: Polydispersity index

PG: Propylene glycol 
CBP: Carbopol

SEM: Scanning electron microscope

HPLC: High-performance liquid chromatography

XRPD: X-ray powder diffraction

FT-IR: Fourier transform infrared spectroscopy

PM: $\quad$ Physical mixture.

\section{Data Availability}

No data were used to support this study.

\section{Ethical Approval}

All institutional and national guidelines for the care and use of laboratory animals were followed.

\section{Conflicts of Interest}

The authors report no conflicts of interest.

\section{Authors' Contributions}

Hoang Nhan Ho and Thien Giap Le contributed equally.

\section{References}

[1] S. Alam, Z. Iqbal, A. Ali et al., "Microemulsion as a potential transdermal carrier for poorly water soluble antifungal drug itraconazole," Journal of Dispersion Science and Technology, vol. 31, no. 1, pp. 84-94, 2009.

[2] A. Chudasama, V. Patel, M. Nivsarkar, K. Vasu, and C. Shishoo, "Investigation of microemulsion system for transdermal delivery of itraconazole," Journal of Advanced Pharmaceutical Technology \& Research, vol. 2, no. 1, pp. 30-38, 2011.

[3] K. K. Mali, S. C. Dhawale, and R. J. Dias, "Microemulsion based bioadhesive gel of itraconazole using tamarind gum: in-vitro and ex-vivo evaluation," Marmara Pharmaceutical Journal, vol. 21, no. 3, pp. 688-688, 2017.

[4] S. El-Housiny, M. A. Shams Eldeen, Y. A. El-Attar et al., "Fluconazole-loaded solid lipid nanoparticles topical gel for treatment of pityriasis versicolor: formulation and clinical study," Drug Delivery, vol. 25, no. 1, pp. 78-90, 2018.

[5] M. Mathur and V. K. Devi, "Potential of novel drug delivery systems in the management of topical candidiasis," Journal of Drug Targeting, vol. 25, no. 8, pp. 685-703, 2017.

[6] Z. Zhang, P. C. Tsai, T. Ramezanli, and B. B. Michniak-Kohn, "Polymeric nanoparticles-based topical delivery systems for the treatment of dermatological diseases," Wiley Interdisciplinary Reviews. Nanomedicine and Nanobiotechnology, vol. 5, no. 3, pp. 205-218, 2013.

[7] G. M. Soliman, "Nanoparticles as safe and effective delivery systems of antifungal agents: achievements and challenges," International Journal of Pharmaceutics, vol. 523, no. 1, pp. 15-32, 2017.

[8] G. S. Rekhi and S. S. Jambhekar, "Ethylcellulose - a polymer review," Drug Development and Industrial Pharmacy, vol. 21, no. 1, pp. 61-77, 2008.

[9] S. Sahu, S. Saraf, C. D. Kaur, and S. Saraf, "Biocompatible nanoparticles for sustained topical delivery of anticancer phytoconstituent quercetin," Pakistan Journal of Biological Sciences, vol. 16, no. 13, pp. 601-609, 2013.
[10] S. Duarah, R. D. Durai, and V. B. Narayanan, "Nanoparticlein-gel system for delivery of vitamin C for topical application," Drug Delivery and Translational Research, vol. 7, no. 5, pp. 750-760, 2017.

[11] B. Balzus, F. F. Sahle, S. Honzke et al., "Formulation and _ex vivo_evaluation of polymeric nanoparticles for controlled delivery of corticosteroids to the skin and the corneal epithelium," European Journal of Pharmaceutics and Biopharmaceutics, vol. 115, pp. 122-130, 2017.

[12] H. T. Nguyen, T. H. Tran, J. O. Kim, C. S. Yong, and C. N. Nguyen, "Enhancing the in vitro anticancer efficacy of artesunate by loading into poly-D,L-lactide-co-glycolide (PLGA) nanoparticles," Archives of Pharmacal Research, vol. 38, no. 5, pp. 716-724, 2015.

[13] C. N. Nguyen, T. T. T. Nguyen, H. T. Nguyen, and T. H. Tran, "Nanostructured lipid carriers to enhance transdermal delivery and efficacy of diclofenac," Drug Delivery and Translational Research, vol. 7, no. 5, pp. 664-673, 2017.

[14] H. N. Ho, I. Laidmae, K. Kogermann et al., "Development of electrosprayed artesunate-loaded core-shell nanoparticles," Drug Development and Industrial Pharmacy, vol. 43, no. 7, pp. 1134-1142, 2017.

[15] X. Ling, Z. Huang, J. Wang et al., "Development of an itraconazole encapsulated polymeric nanoparticle platform for effective antifungal therapy," Journal of Materials Chemistry B, vol. 4, no. 10, pp. 1787-1796, 2016.

[16] M. Yao and N. R. Srinivas, "Bioanalytical methods for the determination of itraconazole and hydroxyitraconazole: overview from clinical pharmacology, pharmacokinetic, pharmacodynamic and metabolism perspectives," Biomedical Chromatography, vol. 23, no. 7, pp. 677-691, 2009.

[17] B. Balzus, M. Colombo, F. F. Sahle, G. Zoubari, S. Staufenbiel, and R. Bodmeier, "Comparison of different_in vitro_release methods used to investigate nanocarriers intended for dermal application," International Journal of Pharmaceutics, vol. 513, no. 1-2, pp. 247-254, 2016.

[18] C. Salerno, A. M. Carlucci, and C. Bregni, "Study of in vitro drug release and percutaneous absorption of fluconazole from topical dosage forms," AAPS PharmSciTech, vol. 11, no. 2, pp. 986-993, 2010.

[19] C. V. Pham, M. C. Van, H. P. Thi et al., "Development of ibuprofen-loaded solid lipid nanoparticle-based hydrogels for enhanced_in vitro_dermal permeation and_in vivo_topical anti-inflammatory activity," Journal of Drug Delivery Science and Technology, vol. 57, p. 101758, 2020.

[20] C. Vitorino, J. Almeida, L. M. Gonçalves, A. J. Almeida, J. J. Sousa, and A. A. Pais, "Co-encapsulating nanostructured lipid carriers for transdermal application: from experimental design to the molecular detail," Journal of Controlled Release, vol. 167, no. 3, pp. 301-314, 2013.

[21] C. López-Castillo, C. Rodríguez-Fernández, M. Córdoba, and J. J. Torrado, "Permeability characteristics of a new antifungal topical amphotericin B formulation with $\gamma$-Cyclodextrins," Molecules, vol. 23, no. 12, p. 3349, 2018.

[22] Y. Liu, G. Tortora, M. E. Ryan, H. M. Lee, and L. M. Golub, "Potato dextrose agar antifungal susceptibility testing for yeasts and molds: evaluation of phosphate effect on antifungal activity of CMT-3," Antimicrobial Agents and Chemotherapy, vol. 46, no. 5, pp. 1455-1461, 2002.

[23] R. M. Mainardes and R. C. Evangelista, "PLGA nanoparticles containing praziquantel: effect of formulation variables on size 
distribution," International Journal of Pharmaceutics, vol. 290, no. 1-2, pp. 137-144, 2005.

[24] L. De Smet, L. Saerens, T. De Beer et al., "Formulation of itraconazole nanococrystals and evaluation of their bioavailability in dogs," European Journal of Pharmaceutics and Biopharmaceutics, vol. 87, no. 1, pp. 107-113, 2014.

[25] T. Mahnaj, S. U. Ahmed, and F. M. Plakogiannis, "Characterization of ethyl cellulose polymer," Pharmaceutical Development and Technology, vol. 18, no. 5, pp. 982-989, 2013.

[26] A. A. Alhowyan, M. A. Altamimi, M. A. Kalam et al., "Antifungal efficacy of itraconazole loaded PLGA-nanoparticles stabilized by vitamin-E TPGS: _in vitro_ and _ex vivo_studies," Journal of Microbiological Methods, vol. 161, pp. 87-95, 2019.

[27] S. E. El-Habashy, A. N. Allam, and A. H. El-Kamel, "Ethyl cellulose nanoparticles as a platform to decrease ulcerogenic potential of piroxicam: formulation and in vitro/in vivo evaluation," International Journal of Nanomedicine, vol. 11, pp. 2369-2380, 2016.

[28] K. Bhaskar, J. Anbu, V. Ravichandiran, V. Venkateswarlu, and Y. M. Rao, "Lipid nanoparticles for transdermal delivery of flurbiprofen: formulation, in vitro, ex vivo and in vivo studies," Lipids in Health and Disease, vol. 8, no. 1, p. 6, 2009.

[29] S. Y. Karavana, E. Gokce, S. Rençber et al., "A new approach to the treatment of recurrent aphthous stomatitis with bioadhesive gels containing cyclosporine A solid lipid nanoparticles: in vivo/in vitro examinations," International Journal of Nanomedicine, vol. 7, pp. 5693-5704, 2012.

[30] I. M. Abdulbaqi, Y. Darwis, R. Abou Assi, and N. Abdul Karim Khan, "Transethosomal gels as carriers for the transdermal delivery of colchicine: statistical optimization, characterization, and ex vivo evaluation," Drug Design, Development and Therapy, vol. Volume 12, pp. 795-813, 2018.

[31] A. H. Alomrani, G. A. Shazly, A. A. Amara, and M. M. Badran, "Itraconazole-hydroxypropyl- $\beta$-cyclodextrin loaded deformable liposomes:_In vitro_skin penetration studies and antifungal efficacy using _Candida albicans_as model," Colloids and Surfaces. B, Biointerfaces, vol. 121, pp. 74-81, 2014.

[32] M. S. Jangdey, C. D. Kaur, and S. Saraf, "Efficacy of Concanavalin-A conjugated nanotransfersomal gel of apigenin for enhanced targeted delivery of UV induced skin malignant melanoma," Artificial Cells, Nanomedicine, and Biotechnology, vol. 47, no. 1, pp. 904-916, 2019.

[33] C. C. Sheth, E. Johnson, M. E. Baker, K. Haynes, and F. A. Muhlschlegel, "Phenotypic identification of Candida albicans by growth on chocolate agar," Medical Mycology, vol. 43, no. 8, pp. 735-738, 2005.

[34] C. Tahir, H. A. Nggada, A. M. Abubakar, M. Garbati, and E. H. Terna Yawe, "Primary cutaneous aspergillosis in an immunocompetent patient," Journal of Surgical Technique and Case Report, vol. 3, no. 2, pp. 94-96, 2011.

[35] J. S. Passos, L. C. de Martino, V. F. C. Dartora, G. L. B. de Araujo, K. Ishida, and L. B. Lopes, "Development, skin targeting and antifungal efficacy of topical lipid nanoparticles containing itraconazole," European Journal of Pharmaceutical Sciences, vol. 149, article 105296, 2020.

[36] N. R. Patel, K. Damann, C. Leonardi, and C. M. Sabliov, "Itraconazole-loaded poly(lactic-co-glycolic) acid nanoparticles for improved antifungal activity," Nanomedicine (London, England), vol. 5, no. 7, pp. 1037-1050, 2010. 